\title{
Flexibilité et sécurité : quelles stratégies d'acteurs
}

Flexibility and security: what strategies?

Jacques Freyssinet

\section{(2) OpenEdition}

Journals

Édition électronique

URL : http://journals.openedition.org/travailemploi/3610

DOI : 10.4000/travailemploi.3610

ISSN : 1775-416X

Éditeur

DARES - Ministère du Travail

Édition imprimée

Date de publication : 5 juin 2009

Pagination : 113-121

ISSN : 0224-4365

\section{Référence électronique}

Jacques Freyssinet, «Flexibilité et sécurité : quelles stratégies d'acteurs », Travail et Emploi [En ligne], 118 | avril-juin 2009, mis en ligne le 03 décembre 2010, consulté le 30 avril 2019. URL : http:// journals.openedition.org/travailemploi/3610; DOI : 10.4000/travailemploi.3610 


\title{
Flexibilité et sécurité: quelles stratégies d'acteurs?
}

\author{
Jacques Freyssinet (*)
}

\section{L'efficacité sociale des "concepts flous"}

L'actualité sociale est périodiquement scandée par l'émergence de notions dont l'usage est aussi fréquent que le contenu est incertain. Le terme de flexicurité en fournit une illustration typique. Deux attitudes sont possibles face à ces innovations langagières:

- une démarche critique rigoureuse peut conduire à les rejeter comme purement idéologiques, voire mystificatrices, du fait de leur indéfinition ou de leurpolysémie;

- la signification de leur apparition dans un contexte historique déterminé peut faire l'objet, comme tout autre phénomène social, d'une analyse objective.

Notre hypothèse est que les «concepts flous» jouent un rôle majeur dans le débat social. Ce sont précisément leur polysémie et leurs ambiguïtés qui permettent que des acteurs ayant des analyses et des objectifs divergents, voire antagonistes, les acceptent comme cadres de référence, au moins provisoires, de leurs négociations.

L'important est donc de comprendre comment se définissent les stratégies des acteurs dans un domaine où le choix du vocabulaire est un élément de la construction des rapports de forces (deuxième point). Une telle interprétation n'est possible que sur la base d'une analyse préalable de la polysémie de manière à mieux comprendre le rôle fonctionnel qu'elle assure dans le débat social (premier point).

\section{Flexibilité et sécurité: sur quel socle fonder l'articulation?}

La conciliation entre des exigences de flexibilité, de mobilité ou d'adaptabilité, d'une part, et des

\footnotetext{
* Professeur émérite à l'université Paris 1 , président du Conseil scientifique du Centre d'études de l'emploi: jacques.freyssinet@cee-recherche.fr
}

garanties de sécurité ou de sécurisation, d'autre part, peut être construite sur plusieurs socles. Pour que cette conciliation soit concevable, il faut adopter une conception de la flexibilité qui renvoie à la capacité d'adaptation du système productif et non à la précarité du statut des travailleurs, ainsi qu'une conception de la sécurité qui s'applique à des trajectoires professionnelles et non à la garantie de l'emploi à vie dans le même métier chez le même employeur. Dans ce cadre, l'évolution historique des marchés $\mathrm{du}$ travail et les théories économiques qui en ont fourni des modélisations montrent que l'articulation entre flexibilité productive et sécurité professionnelle peut être construite sur plusieurs socles. Les trois formes principales de mise en œuvre reposent sur le contrat de travail, la qualification (ou les compétences) et le territoire (FREYSSINET, 2006).

\section{Le contrat de travail}

Dans cette première perspective, le compromis flexibilité/sécurité s'établit sur la base du lien juridique qui unit le salarié à l'employeur dans le cadre de normes d'origine légale, conventionnelle ou coutumière. Les marchés internes du travail fournissent le modèle de référence (1) en combinant des garanties de durabilité de la relation d'emploi, des espaces internes de mobilité ascendante et des modes de construction de qualifications spécifiques. Ce modèle se heurte aujourd'hui à des obstacles et à des critiques majeurs.

En premier lieu, ses conditions d'efficacité (prévisibilité d'un niveau d'activité, stable ou croissant, adaptabilité du collectif de travail aux changements techniques et organisationnels) sont de plus en plus rarement réunies ou ne le sont que pour des segments décroissants de la force de travail.

En second lieu, la référence au contrat de travail donne un rôle déterminant à l'identification de l'employeur au sens juridique du terme. De multiples innovations juridiques et gestionnaires ont fait que celui-ci

(1) Le point de départ est fourni par l'ouvrage de Peter DoERINGER et Michael PIORE (1971). 
est de plus en plus souvent distinct de l'utilisateur effectif de la force de travail(2). La question se pose de savoir sur qui faire peser les obligations de sécurisation: l'entreprise, le groupe, le donneur d'ordres...?

Enfin et surtout, un débat porte sur la nature de l'obligation de l'entreprise. La sécurité dans la flexibilité peut être assurée, selon par exemple l'orientation actuelle de la loi et de la jurisprudence en France, au moyen d'obligations de faire (maintenir l'employabilité, assurer le reclassement interne ou externe...); ces garanties sont encadrées par des procédures de consultation et/ou de négociation. On peut alternativement considérer que la sécurité dans la flexibilité serait garantie par l'obligation de payer faite à l'entreprise, au prorata des déséconomies externes qu'elle engendre par ses licenciements (3). La différence est considérable puisque, dans le premier cas, on fait appel à des mécanismes collectifs fonctionnant sous le contrôle du juge et, dans le second, à des incitations financières qui visent à infléchir le calcul économique d'un employeur libre de ses décisions (GAUTIÉ, 2006).

\section{La qualification ou les compétences}

Le compromis flexibilité/sécurité repose, dans une seconde perspective, sur l'autonomie du salarié à l'égard de l'employeur et sur la capacité du premier à maîtriser ou à négocier sa trajectoire professionnelle. Il existe deux variantes contrastées de ce modèle.

\section{Craft markets et marchés professionnels}

Ces marchés ont souvent été analysés (4). Ils impliquent des institutions (syndicales ou paritaires) de production et de validation de qualifications transférables, complétées éventuellement par des mécanismes de régulation des marchés du travail.

Les facteurs de leur crise sont connus: cloisonnement, ou «balkanisation», des marchés du travail, faible ou lente capacité d'adaptation aux change-

(2) Externalisation et sous-traitance, diverses formes de prêt ou de mise à disposition de main-d'œuvre, etc.

(3) Deux mécanismes différents ont été proposés selon cette logique. Olivier Blanchard et Jean Tirole combinent d'une part, une taxe de licenciement, versée à la caisse d'assurance chômage et proportionnelle au nombre de licenciements, d'autre part, des indemnités de licenciement versées au salarié et proportionnelles à son ancienneté (Blanchard, Tirole, 2003). Pierre Cahuc et Francis Kramarz envisagent également une indemnité de licenciement versée au salarié, mais ils la combinent avec une contribution de solidarité versée au service public de l'emploi pour financer les opérations de réinsertion des travailleurs licenciés. Ces deux composantes ont un montant proportionnel à la rémunération totale perçue depuis l'embauche, avec un supplément pendant les dix-huit premiers mois pour l'indemnité de licenciement (Cahuc, Kramarz, 2005).

(4) C'est probablement à Clark KerR (1954) que l'on doit la première formalisation théorique des craft markets. David MARSDEN (1989) a proposé plus tard un élargissement de cette approche à partir de la notion de marché professionnel. ments techniques, prime accordée aux stratégies de free riders.

Cependant, on doit se demander si les formes d'organisation des professions (au sens anglo-saxon du terme), longtemps limitées aux professions libérales, ne proposent pas aujourd'hui un modèle de flexibilité/sécurité en voie d'expansion pour de nombreuses catégories de salariés qualifiés (5). La certification et la transférabilité des qualifications, garanties par des règles collectives issues de la loi ou de la négociation, fournissent le socle d'une conciliation entre mobilité et sécurité.

\section{Le salarié responsable de son employabilité}

Un second modèle, qui privilégie la notion de compétences, propose une formalisation qui s'insère dans la conception néoclassique du marché du travail. Le travailleur assure la sécurité de sa trajectoire professionnelle grâce à une stratégie d'apprentissage tout au long de la vie qui développe son capital humain et garantit son employabilité.

Deux difficultés principales apparaissent: -il s'agit d'une version appauvrie de la théorie du capital humain «à la Becker» (BECKER, 1964) puisque l'existence de qualifications spécifiques est ici ignorée. Les prendre en compte supposerait une reconnaissance de la responsabilité de l'entreprise dans leur production;

- face aux risques de sous optimalité des dépenses de formation et d'amplification des inégalités sociales, les solutions proposées (subventions, accès au crédit, droits de tirage, vouchers...) négligent le fait que l'apprentissage et, plus fondamentalement, la demande de formation dépendent de la qualité du travail et de la mise en place d'une organisation $\mathrm{du}$ travail qualifiante (learning organization). Ici encore, les choix de l'entreprise contribuent fortement à déterminer l'employabilité.

La logique de la qualification, ou des compétences, n'est donc pas unifiée. Comme dans le modèle précédent, il apparaît qu'elle peut se traduire par la mise en place de règles et d'institutions, issues de processus de concertation ou de négociation et au sein desquelles s'exercent des choix individuels. Alternativement, elle peut s'appuyer uniquement sur le calcul microéconomique, infléchi par des incitations financières, positives ou négatives, de la puissance publique.

\section{Le territoire}

La référence au territoire comme socle d'un compromis entre flexibilité et sécurité est à la fois faiblement construite théoriquement et fortement

(5) Dans le cas de la France, voir l'ouvrage de Claude Dubar et Pierre TrIPIER (1998) à propos de l'extension et de la diversification des professions. 
présente dans les préoccupations des acteurs. Depuis la première analyse des «districts industriels» (MARSHALl, 1898) jusqu'à leur redécouverte sous la forme des «districts italiens», puis des «systèmes productifs territorialisés », ont été mis en évidence des mécanismes de sécurité de l'emploi assurés par de multiples mobilités locales conjointes (entre statuts, entre entreprises, entre activités...).

Divers facteurs contribuent à renforcer l'importance accordée à cette approche :

-1 'accroissement de l'attachement des personnes à leur territoire d'appartenance (ou d'élection) et les contraintes qui réduisent la mobilité géographique (logement, couples à deux actifs...);

- la décentralisation des compétences politiques et la mobilisation des acteurs locaux sur les objectifs d'emploi;

- les fragilités engendrées par la dépendance d'un territoire à l'égard d'un employeur dominant.

Les «pactes territoriaux pour l'emploi» de l'Union européenne fournissent de multiples illustrations de cette démarche encore largement empirique. L'un des enseignements apportés par l'étude d'expériences récentes (JOBERT, 2008; VERDIER, 2008) est que les réussites, toujours fragiles, reposent sur la coopération d'acteurs nombreux et hétérogènes qui, au-delà de leurs différences de nature et de leurs divergences d'intérêts, parviennent à s'accorder sur des projets retenant la qualité de l'emploi et du travail comme objectif partagé.

La volonté de distinguer entre les différents socles qui peuvent permettre de construire un lien entre flexibilité productive et sécurité professionnelle répond à un souci analytique. Elle n'a pas pour objet de mettre en demeure les acteurs de choisir entre ces différentes conceptions. Il est parfaitement possible d'envisager de les développer de manière conjointe, comme le fait, par exemple l'approche des «marchés transitionnels» (GAZIER, 2003 et 2008). Encore importe-t-il d'approfondir l'examen des relations de complémentarité ou de contradiction qui peuvent s'établir entre elles et des jeux d'avantages et de concessions réciproques qui peuvent s'organiser autour d'elles. Nous entrons dans le domaine de la stratégie des acteurs.

\section{Flexibilité et sécurité: stratégies des acteurs}

L'approche devient plus délicate puisqu'il s'agit de mesurer ce que le choix d'un langage et des exemples qui servent à l'illustrer révèle quant aux stratégies des acteurs, aux domaines de compromis possibles et à la nature des enjeux ouverts.

\section{État des lieux}

La pertinence, pour la France, de la réévaluation de la Stratégie de l'OCDE pour l'emploi doit être située dans un processus qui, tant au niveau international que national, mobilise de multiples acteurs. Avec un risque d'arbitraire, nous retenons certains d'entre eux qui ont joué un rôle, intellectuel ou politique, particulièrement décisif.

\section{Les organisations internationales: OCDE et Union européenne}

Le message de ces deux institutions s'exprime directement par les recommandations ou lignes directrices qu'elles adoptent et indirectement au travers des «bonnes pratiques» qu'elles sélectionnent pour illustrer leurs analyses.

Dans le domaine des recommandations, le contraste est clair, au départ, entre les discours des deux organisations (encadré 1). L'OCDE privilégie la flexibilité (6) alors que l'Union européenne emploie un langage d'équilibre.

Encadré 1
OCDE - Stratégie pour l'emploi (1994)
Dix grandes recommandations
(extraits)
3) Accroitre la flexibilité du temps de travail
[...].
5) Accroitre la flexibilité des coûts salariaux
et de main-d'œuvre [...].
6) Revoir les dispositions relatives à la sécu-
rité de l'emploi qui freinent l'expansion de l'em-
ploi [...].
Union européenne
Les premières «lignes directrices" (1998)
(extraits)
[...] moderniser l'organisation du travail [...]
afin de rendre les entreprises productives et
compétitives et d'atteindre l'équilibre néces-
saire entre souplesse et sécurité.
[...] introduire des contrats plus adaptables
[...]. Les personnes travaillant dans le cadre de
contrats de ce type devraient, dans le même
temps, bénéficier d'une sécurité suffisante et
d'un meilleur statut professionnel, compatible
avec les nécessités des entreprises.

Après la Jobs Study (OCDE, 1994), les Études économiques de l'OCDE déclineront inlassablement les recommandations pour chaque pays en

(6) Il est remarquable qu'au sein des dix grandes recommandations de la Stratégie pour l'emploi, en 1994, le mot "sécurité» n'est utilisé qu'en référence aux freins que cette dernière peut constituer pour l'expansion de l'emploi. 
donnant clairement la priorité à la flexibilité (7). À l'opposé, les textes de l'Union européenne conservent des formulations équilibrées; ils puisent une part de leur argumentation dans divers rapports qui ont eu une forte résonance, par exemple ceux réalisés sous la responsabilité d'Alain SuPIOT et de Wim KoK (SUPIOT, 1999; KoK, 2003). On retrouve le même esprit dans le récent Livre vert sur le droit du travail (COMMISSION EUROPÉENNE, 2006a).

La distance semble aujourd'hui considérablement réduite entre le langage des deux institutions (8). On observe, en particulier (encadré 2), que, pour le temps de travail et les salaires, les termes d' «adaptation » et d' "aménagement» ont remplacé celui de «flexibilité» dans le langage de l'OCDE (9).

\section{Encadré 2 \\ Union européenne Lignes directrices intégrées pour la croissance et l'emploi (2005-2008) (extraits)}

(21) Favoriser la fl exibilité en la conciliant avec la sécurité de l'emploi et réduire la segmentation de marché du travail, en tenant dûment compte du rôle des partenaires sociaux.

\section{OCDE}

\section{Stratégie pour l'emploi révisée (2006) (extraits) Pilier C}

- Faire en sorte que les salaires et les coûts de main-d'œuvre s'adaptent aux évolutions du marché du travail.

- Faciliter l'adoption de dispositifs d'aménagement du temps de travail.

- Faire en sorte que la législation sur la protection de l'emploi contribue au dynamisme du marché du travail et assure la sécurité des travailleurs.

Si l'on s'intéresse, maintenant, aux «bonnes pratiques », il est significatif de repérer les exemples, présentés comme positifs, qui illustrent les analyses des deux institutions (par exemple, dans OCDE, 2006a et COMMISSION EUROPÉENNE, 2006b, chapitre $2)$. Une analyse textuelle systématique serait nécessaire; nous nous limiterons à quelques illustrations. - Les Pays-Bas sont généralement désignés comme le pays d'origine de la flexicurité. Les références portent, d'une part, sur la législation ayant élargi les conditions de recours aux formes d'emploi atypiques

(7) Récemment encore, le Département des affaires économiques a appliqué cette approche au cas de la France, JAMET, 2006.

(8) Sur le lien complexe et évolutif entre les analyses de l'OCDE et celles de la Commission européenne, voir la pénétrante analyse de Niclos NOAKSSON et Kerstin JACOBSSON, 2003. (9) Inflexion sur le fond ou simple habileté sémantique (WATT, 2006)? Le débat est ouvert, mais notre hypothèse est que le choix du langage a toujours un sens. en échange du renforcement des garanties qui leur sont attachées, d'autre part, sur le droit reconnu aux salariés de demander à leur employeur une augmentation ou une diminution de leur durée de travail.

- Le Danemark est devenu la référence à la mode. L'accent est mis sur la cohérence qui y a été construite entre le faible degré de législation protectrice de l'emploi (LPE), le niveau élevé d'indemnisation du chômage et l'importance des dépenses actives de politique de l'emploi (suivi individualisé).

- L'Autriche, longtemps citée au titre des Fondations du travail (responsabilité des entreprises dans la reconversion des travailleurs licenciés), l'est maintenant pour la création d'un fonds de mutualisation des indemnités de licenciement transformées en comptes individuels.

-L'Espagne est mentionnée au titre des accords tripartites de 1997 et 2006 qui échangent un usage plus limité et mieux contrôlé des contrats précaires contre la création d'un nouveau type de contrat de travail à durée indéterminée, avec de plus faibles indemnités en cas de licenciement économique.

Ce début d'inventaire pourrait facilement être enrichi(10). Il illustre l'extrême diversité des champs couverts par la notion de flexicurité. Elle désigne plus une orientation générale innovatrice qu'un domaine spécifique de la relation d'emploi.

\section{L'ouverture du débat en France: experts et syndicats}

Même si le Gouvernement et les partis politiques se sont récemment emparés du thème, l'élan initial provient principalement, en France, de deux sources distinctes.

En premier lieu, plusieurs rapports ont alimenté les débats publics, depuis celui de Jean Boissonnat avec, notamment, le projet de contrat d'activité, jusqu'à celui de Pierre Cahuc et Francis Kramarz sur la sécurité sociale professionnelle (11).

En second lieu, les confédérations syndicales, avec des vocabulaires distincts, mais voisins ont explicité leurs revendications de sécurité dans les mobilités professionnelles (12):

-CGT: «sécurité sociale professionnelle» dans le cadre d'un «nouveau statut du travail salarié»;

-CFDT: «sécurisation des parcours professionnels» dans le cadre d'une "rénovation du contrat de travail»;

-CFTC: «sécuriser les parcours de vie»;

(10) Voir, par exemple, AuER, GAZIER, 2006

(11) Boissonnat, 1995; Cahuc, Kramarz, 2004. Voir aussi, plus récemment, les rapports du Conseil de l'emploi des revenus et de la cohésion sociale (CERC, 2005), du Conseil d'orientation pour l'emploi (COE, 2007) et du Conseil économique et social (ARNoult-BriLl, 2007). Pour des approches synthétiques: Duclos, Kerbourc'h, 2006; Méda, Minault, 2005.

(12) Pour une analyse comparative, avec un essai stimulant de cartographie, voir GrIMAULt, 2008. 
-CFE-CGC: «assurance emploi» dans le cadre d'un accord national interprofessionnel sur «la sécurité d ans 1 'emploi»;

-UNSA: «droit à la sécurité et à la progression professionnelles» (13).

S'il n'existe pas de prise de position globale du MEDEF ou des autres organisations patronales sur la flexicurité et si leurs demandes ont jusqu'ici porté essentiellement sur la seule flexibilité (MEDEF, 2004 et 2007), c'est le MEDEF qui a pris, en octobre 2006, l'initiative de discussions avec les syndicats sur trois thèmes directement liés: les contrats de travail (contrat unique), la sécurisation des parcours professionnels, la «remise à plat» de l'assurance chômage. La juxtaposition des thèmes ne pouvait alors cacher leur inéluctable interdépendance.

\section{Quelques problèmes}

L'évolution des problématiques des acteurs et l'accumulation d'expériences partielles montrent que certaines conditions étaient réunies pour l'ouverture d'une négociation plus globale. En raisonnant sur le cas de la France, il est possible d'identifier quelques enjeux qui en conditionnent les progrès.

\section{Complémentarités et contradictions}

Il est logique que les acteurs ne dévoilent pas, au départ, leurs choix quant aux arbitrages qu'ils devront faire au cours de la négociation entre les différents objectifs qu'ils poursuivent. Il est cependant utile de mettre en évidence les risques de contradictions internes auxquels ils sont confrontés.

Du côté syndical, la proximité est forte en ce qui concerne les principaux instruments de sécurisation revendiqués par les différentes organisations : gestion prévisionnelle de l'emploi et observatoires des métiers, accès de tous les salariés à une formation continue qualifiante, garanties sur les procédures de licenciement et les dispositifs de reconversion, accompagnement individualisé des demandeurs d'emploi, mutualisation des financements... Il reste à préciser sur la base de quelle logique dominante cet ensemble de moyens est mis en cohérence. La principale difficulté réside peut-être dans l'articulation entre les deux objectifs simultanément affirmés de renforcement de la responsabilité sociale de l'entreprise et de l'autonomie des salariés. Limitonsnous aux cas des deux organisations ayant sur ce point les positions les plus contrastées.

(13) La CGT-FO est méfiante à l'égard de ce vocabulaire. Cependant, à l'occasion de la première rencontre des partenaires sociaux sur la réforme du marché du travail (23 octobre 2006), on peut lire la phrase suivante dans l'intervention de son secrétaire général, Jean-Claude Mailly: «La démarche doit viser à contrôler, anticiper, juguler et réglementer par la création de normes, ce qui tend à opposer la fluidité à la flexibilité». Si la priorité est donnée à la production de normes, c'est dans le cadre d'une fluidité acceptée qui est opposée à la flexibilité.
Les dirigeants de la CGT ont affirmé l'importance qu'ils attachaient au second aspect. Par exemple Jean-Christophe Le Duigou, secrétaire confédéral, demande «la reconnaissance d'un droit à la mobilité professionnelle, à l'organisation d'une carrière diversifiée, à la définition de formes de travail complémentaires» (Le Duigou, 2005, p. 48). Maryse Dumas, secrétaire confédérale, revendique «un droit de l'individu tout au long de sa vie qui le libère de sa dépendance au devenir et à la gestion de telle ou telle entreprise» (14). Pour Bernard Thibault, secrétaire général, «il s'agit d'accrô̂tre la mobilité professionnelle dans la liberté, c'est-à-dire la capacité personnelle à s'orienter professionnellement»(15). Mais, dans le même temps, la CGT considère qu'en cas de licenciement, «le contrat de travail et la rémunération doivent être maintenus jusqu'à ce que le salarié ait obtenu un reclassement effectif dans un nouvel emploi de niveau au moins équivalent au précédent» (16).

Sur ce point, la CFDT affirme son désaccord: «La CFDT n'entend pas sécuriser le contrat, mais bien les trajectoires professionnelles. Il n'est pas question de surprotéger le salarié, mais de faire en sorte qu'il soit acteur de son parcours» (PIRAT, 2006, p. 1). L'objectif, selon Annie Thomas, secrétaire nationale, est de "négocier collectivement de nouveaux droits pour accompagner ces mobilités et ces transitions » (ibidem). Cependant, il est aussi affirmé que «la sécurisation des parcours professionnels s'inscrit dans la responsabilité sociale des entreprises et des fonctions publiques » (17), c'està-dire des employeurs.

Une voie de réconciliation entre les deux approches réside dans une mutualisation du financement de l'exercice de ces droits, solution soutenue par les deux organisations. Mais alors apparaît la question du champ dans lequel s'organiserait la solidarité: la branche ou le territoire? Les deux solutions sont évoquées, sans préférence explicite.

Nous reconnaissons ici les trois socles possibles de construction des droits permettant de garantir une mobilité dans la sécurité (18): la responsabilité sociale de l'employeur, l'autonomie d'un salarié aux qualifications transférables, les complémentarités et solidarités dans les territoires. Les trois voies peuvent être explorées parallèlement, mais il

(14) Introduction à la Commission exécutive confédérale du 6 décembre 2005: www.cgt.fr

(15) Entretien accordé à Vacarme, automne 2005.

(16) Point $2 \mathrm{P} 022$ de la résolution du congrès confédéral: www. cgt.fr

(17) Laurence Laigo, «La sécurisation des parcours professionnels. Perspectives», bureau national CFDT, 15-16 novembre 2006.

(18) Pour faire bref, nous n'avons évoqué que la position de deux organisations. Le même exercice pourrait naturellement être réalisé pour les autres. Voir la contribution, déjà citée, de Solveig Grimault (2008). 
existera un moment où des arbitrages seront nécessaires pour assurer la cohérence.

$\mathrm{Du}$ côté patronal, une contradiction potentielle résulte de la demande simultanée d'une «sécurisation juridique de l'employeur» et d'un retour aux principes du droit civil dans la gestion du contrat individuel de travail. Réagissant au rapport présenté par Michel de Virville (VIRVILLE, 2004), auquel il «adhère globalement», le MEDEF a rendu publiques ses propres propositions (MEDEF, 2004). D'entrée, certains objectifs sont mis en avant.

- «Redonner toute sa force au contrat, individuel et collectif $[\ldots]$.

- Réaffirmer l'application des principes généraux du droit civil au contrat de travail [...].

- Rétablir dans tous les cas l'application de la règle qui impose au demandeur d'apporter la preuve de ses allégations et bannir l'existence d'une preuve négative à la charge du défendeur [...].

- Créer un bloc de compétence pour que les litiges nés à l'occasion de l'application du droit du travail relèvent exclusivement de la compétence judiciaire»(op. cit., p. 4).

Cette démarche est présentée comme une réaction contre la prolifération de dispositions dérogatoires du droit commun, progressivement sédimentées dans le Code du travail (GAudu, 2006). Sans en discuter sur le fond, nous entendons seulement examiner son degré de cohérence avec la revendication de sécurisation juridique. Deux rappels historiques permettent d'amorcer la réflexion.

Les accidents du travail ont été, durant les trois premiers quarts du $\mathrm{XIX}^{\mathrm{e}}$ siècle, régis par les principes du droit civil: règles de responsabilité, charge de la preuve, délai de recours... Les travaux de François Ewald montrent que le développement d'institutions patronales dans ce domaine a d'abord eu pour but, en ce qui concerne les employeurs, «d'échapper à la sanction juridique de leurs obligations, d'éviter l'immixtion du judiciaire» (EwALD, 1996, p. 74). L'extension progressive par la jurisprudence du champ de la responsabilité patronale en cas d'accident du travail multiplie les bases de l'action judiciaire des salariés, aiguise les manifestations de l'antagonisme capital-travail, accroît l'incertitude pour les employeurs dans l'attente des décisions des juridictions civiles et pénales. Après dix-huit années de débat, le compromis adopté dans la loi de 1898 rompt avec les principes du droit civil: la réparation intégrale et individualisée, fonction du préjudice et de l'appréciation des responsabilités, est remplacée par une réparation forfaitaire associée à la notion de risque professionnel et garantie au salarié, sauf «faute inexcusable» de sa part. C'est bien un régime d'exception, étranger à la logique du contrat et de la responsabilité issue du droit privé, qui assure la sécurisation pour les deux parties: pour l'employeur, coûts mutualisés selon un barème; pour le salarié, certitude d'une indemnisation en fonction du taux d'incapacité. Ainsi les accidents du travail cessent-ils d'être une cause permanente de conflits dans l'entreprise et de «judiciarisation» des relations d'emploi. François Ewald fait de cette loi l'acte de naissance d'un droit du travail distinct du droit commun et non simplement complémentaire de celui-ci.

La suppression de l'autorisation administrative des licenciements économiques, en 1986, est présentée, à l'époque, comme la réponse symbolique que donne une nouvelle majorité parlementaire de droite aux demandes répétées du patronat. Elle fait disparaître un régime d'exception qui constituait une entrave à la liberté de gestion des employeurs. L'expérience est trop connue pour qu'il soit utile de la rappeler dans le détail.

L'autorisation administrative était, in fine, presque toujours accordée, mais au terme d'une négociation entre l'entreprise et l'Inspection du travail, négociation qui portait conjointement sur le nombre des licenciements, la qualité du plan social et les contreparties apportées par l'État grâce, en particulier, aux crédits du Fonds national de l'emploi (FNE). L'inspecteur du travail était, au cours de cette négociation, en contact régulier avec les représentants des salariés. Cette procédure engendrait un délai pour l'entreprise, mais lui apportait, outre des aides publiques, une sécurité juridique puisque les tribunaux considéraient que l'autorisation administrative établissait la légitimité de la décision de licenciement.

La suppression de l'autorisation administrative et le caractère purement consultatif de l'intervention des instances de représentation du personnel font que la décision de licenciement ne peut être contestée que devant les tribunaux. Aux discussions tripartites entre des acteurs qui parlaient le même langage, se substitue une controverse juridique dans le prétoire. Au cas par cas, la chambre sociale de la Cour de cassation construit progressivement une jurisprudence originale qui est, à diverses reprises, validée ou infléchie par la loi.

C'est clairement le triomphe d'une revendication du patronat, visant au recul de l'intervention publique dans la relation d'emploi, qui a poussé à la «judiciarisation» du traitement des licenciements économiques et engendré une longue période d'insécurité juridique, c'est-à-dire d'incertitude pour l'employeur (comme pour les salariés) sur la nature de la décision finale, le délai dans lequel elle sera rendue et les coûts financiers qui en résulteront.

Associer restauration de la primauté du contrat de travail individuel et sécurisation juridique de l'employeur apparait donc comme un contresens. Par nature, le contrat individuel de droit privé est incomplet parce qu'il ne peut prévoir l'intégralité des avenirs possibles(19). La relation d'emploi

(19) Sauf le cas limite d'un contrat spot portant sur une marchandise parfaitement normalisée. 
fournit, dans le cas du contrat de travail, une illustration typique de l'incomplétude des contrats. Plus les normes collectives (publiques ou conventionnelles) sont réduites, plus large est, en cas de litige, le pouvoir d'appréciation du juge qui applique au cas d'espèce les principes généraux du droit privé, notamment le droit des contrats et le droit de la responsabilité. Plus forte est alors pour les cocontractants l'incertitude sur l'issue du procès. De même, plus réduites sont les règles et les procédures collectives de règlement des conflits et plus forte est la propension à la «judiciarisation» (20). Historiquement, l'adoption de normes générales s'imposant aux contrats de travail a eu, entre autres fonctions, celle de réduire l'incertitude juridique pour les deux parties au contrat et ceci d'autant mieux que les normes étaient plus stables et plus précises (21). Détruire de telles normes, ou les réduire à de simples principes généraux, c'est créer l'insécurité juridique. Le patronat doit choisir entre deux revendications. En revanche, le débat est ouvert sur la nature des normes, publiques ou conventionnelles, et sur leur territoire de référence, depuis l'Europe jusqu'au niveau le plus décentralisé.

\section{Une négociation multidimensionnelle de moyen terme?}

Rendre compatible la flexibilité productive et la sécurité des trajectoires exige, au niveau de l'entreprise, de multiples transformations conjointes qui s'étalent dans le temps : organisation du travail qualifiante, formation tout au long de la vie, gestion prévisionnelle de l'emploi et des compétences, aménagement du temps de travail, salaires et classifications, méthodes de gestion des restructurations... Sur ces thèmes des négociations à l'échelle interprofessionnelle et à celle de la branche sont indispensables, mais il ne peut s'agir que de définir des orientations générales, des règles de procédure et, le cas échéant, des dispositifs de mutualisation(22). En revanche,

(20) Il est remarquable qu'au nom de la «sécurisation juridique» de l'employeur, aient été introduites dans le défunt CNE (contrat nouvelle embauche), puis dans le CPE (contrat première embauche) mort-né des dispositions qui étaient en contradiction avec la logique traditionnelle des contrats, par exemple, la prescription au bout de douze mois de toute contestation sur la rupture.

(21) En ce sens, il est vrai que des normes multiples, instables et parfois contradictoires sont créatrices d'une insécurité juridique qu'il convient de réduire au minimum.

(22) L'échec des négociations sur la flexibilité en 1984 avait durablement condamné la perspective de négociations multidimensionnelles au niveau interprofessionnel. Sous cet aspect, l'accord du 11 janvier 2008 sur la modernisation du marché du travail et les trois accords d'application négociés fin 2008 (gestion prévisionnelle de l'emploi et des compétences, assurance chômage, formation professionnelle tout au long de la vie) marquent, quel que soit le jugement porté sur leur contenu, la volonté des acteurs sociaux de prendre en compte dans leur globalité les transformations de la gestion de l'emploi et du fonctionnement des marchés du travail (FrEYssinet, 2007). ce n'est qu'à l'échelle de l'entreprise (23) qu'il est possible d'assurer, par une négociation multidimensionnelle, d'abord la mise en cohérence des dispositifs et des calendriers, ensuite et peut-être surtout, la mise en équivalence des gains et des concessions de chaque partie, avec une durée d'application qui rende possible des évaluations et des ajustements.

Dans l'expérience française contemporaine, ce type de négociations soulève, la méfiance des acteurs :

- le patronat craint d'être entraîné dans une mise en discussion de la stratégie de l'entreprise, ce qu'il rejette par principe;

- dans le médiocre contexte du marché du travail, les syndicats craignent un rapport des forces défavorable qui les entraînerait dans une logique de concession bargaining à l'Américaine (24); ils s'estiment souvent mieux armés pour négocier séparément sur des thèmes délimités.

L'acceptation du principe de négociations multidimensionnelles de moyen terme au niveau des entreprises paraît être une condition nécessaire pour traiter, à ce niveau, l'articulation entre flexibilité productive et sécurité professionnelle.

\section{Négociations de contreparties et représentativité}

Quelles que soient les positions de départ, aucun des acteurs n'imagine qu'un accord puisse être trouvé en matière de flexicurité sans des concessions réciproques, inévitablement coûteuses. Elles peuvent engendrer, de part et d'autre, des réactions négatives soit au sein des organisations concernées, soit dans les catégories sociales qu'elles représentent. La crédibilité d'un accord est conditionnée par la conviction, chez chacun des signataires, que son interlocuteur a la volonté et la capacité de faire accepter et appliquer le compromis. Ceci suppose la légitimité de celui qui signe l'accord aux yeux de ceux auxquels l'accord s'applique (au moins, de la majorité d'entre eux). Ce n'est pas le lieu ici d'examiner les problèmes de la représentativité des acteurs sociaux (25), mais il est nécessaire d'affirmer qu'il doit être traité au fond si l'on veut rendre possibles des progrès négociés, significatifs et durables, dans la complexe et conflictuelle articulation de la flexibilité productive et de la sécurité professionnelle.

(23) Avec les difficultés signalées plus haut quant à la définition du niveau pertinent: société, groupe de sociétés, donneur d'ordre et sous-traitants...

(24) Abandon de droits acquis dans l'espoir de préserver l'emploi.

(25) $\mathrm{La}$ «position commune» du 9 avril 2009 et la loi du 20 août 2008 ont mis en évidence la profondeur des désaccords sur ce point au sein des organisations syndicales comme patronales. 


\section{Bibliographie}

Arnoult-Brill E. (2007), La sécurisation des parcours professionnels, Conseil économique et social, avis et rapports.

Auer P., Gazier B. (2006), L’introuvable sécurité de l'emploi, Paris, Flammarion.

Becker G.S. (1964), Human Capital. A theoretical and empirical analysis with special reference to education, New York, NBER.

Blanchard O., Tirole J. (2003), Protection de l'emploi et procédures de licenciement, rapport au Conseil d'analyse économique, Paris, La Documentation française.

BoissonnAT J. (rapport de la commission présidée par) (1995), Le travail dans vingt ans, Commissariat général du Plan, Paris, Odile Jacob.

Cahuc P., Kramarz F. (2005), De la précarité à la mobilité: vers une sécurité sociale professionnelle, Paris, La Documentation française.

CERC (Conseil de l'emploi, des revenus et de la cohésion sociale) (2005), La sécurité de l'emploi face aux défis des transformations économiques, Paris, La Documentation française.

CoE (Conseil d'orientation pour l'emploi) (2007), Rapport d'étape sur la sécurisation et la dynamisation des parcours professionnels, Paris.

Commission européEnne (2006a), Moderniser le droit du travail pour relever les défis du XX siècle, Livre vert, Bruxelles.

COMMISSION EUROPÉENNE (2006b), L'emploi en Europe 2006, chapitre 2 «Flexibilité et sécurité dans les marchés du travail de l'Union européenne», Luxembourg.

Doeringer P.B., Piore M.J. (1971), Internal Labor Markets and Manpower Analysis (2 ${ }^{\mathrm{e}}$ ed., 1985), New York, M.E. Sharpe.

Dubar C., Tripier P. (1998), Sociologie des professions, Paris, Armand Colin (réédition 2003).

Duclos L., Kerbourc'h J.-Y. (2006), Organisation du marché du travail et "flexicurité» à la française, CAS, document de travail.

Ewald F. (1996), Histoire de l'État providence, Paris, Grasset.

FREYSSINET J. (2006), «Quels acteurs et quels niveaux pertinents de représentation dans un système productif en restructuration?», p. 213-234 in H. Petit, N. Thévenot (sous la direction de), Les nouvelles frontières du travail subordonné, Paris, La Découverte.

Freyssinet J. (2007), «L'accord du 11 janvier 2008 sur la modernisation du marché du travail: un avenir incertain », La revue de l'IRES, n 54, p. 3-40.

GAudu F. (2006), «Libéralisation des marchés et droit du travail», Droit social, $\mathrm{n}^{\circ}$ 5, p. 505-513.
GAutié G. (2006), «Faut-il taxer plutôt que réglementer les licenciements?», Connaissance de l'emploi, $\mathrm{n}^{\circ} 5$, Centre d'études de l'emploi.

Gazier B. (2003), Tous «Sublimes». Vers un nouveau plein-emploi, Paris, Flammarion.

GAZIER B. (2008), «Flexibilité et marchés transitionnels du travail: esquisse d'une réflexion normative, Travail et Emploi, $\mathrm{n}^{\circ} 113$, p. 117-128.

Grimault S. (2008), «Sécurisation des parcours professionnels et flexicurité : analyse comparative des positions syndicales », Travail et Emploi, n 113, p. 77-91.

Jamet St. (2006), Améliorer la performance du marché du travail en France, OCDE, département des affaires économiques, document de travail n ${ }^{\circ} 504$.

Jobert A. (sous la direction de) (2008), Les nouveaux cadres du dialogue social. Europe et territoires, Bruxelles, PIE Peter Lang.

KerR C. (1954), “The Balkanization of Labor Markets", p. 92-110 in: E.W. Bakke (ed.), Labor Mobility and Economic Opportunity, Cambridge, MIT Press.

KoK W. (Report of the Employment Taskforce chaired by) (2003), Jobs, Jobs, Jobs. Creating more employment in Europe, European Union, Brussels.

Le Duigou J.-C. (2005), «La sécurité sociale professionnelle. Une utopie réaliste», Analyses et documents économiques, $\mathrm{n}^{\circ}$ 98, p. 44-49.

Marsden D. (1989), Marchés du travail. Limites sociales des nouvelles théories, Paris, Economica.

Marshall A. (1898), Principes d'économie politique, Livre IV, chapitre X. «Concentration d'industries spécialisées dans certaines localités», Gordon \& Breach, Paris (rééd. 1971).

Méda D., Minault B. (2005), La sécurisation des trajectoires professionnelles, DARES, document d'études, $\mathrm{n}^{\circ} 107$.

Medef (2004), Moderniser le Code du travail: les 44 propositions du MEDEF, Paris.

Medef (sous la direction de Laurence Parisot) (2007), Besoin d'air, Paris, éditions du Seuil.

NoAksson N., Jacobsson K. (2003), The Production of Ideas and Expert Knowledge in OECD. The OECD Job Strategy in Contrast with the EU Employment Strategy, Score Rapport serie 2003.7, Stockholm.

OCDE (1994), Étude de l'OCDE sur l'emploi, Paris.

OCDE (2006a), Perspectives de l'emploi de l'OCDE. Stimuler l'emploi et les revenus, disponible sur le site: http://www.sourceocde.org/emploi/9264023860

OCDE (2006b), Stimuler l'emploi et les revenus. Les leçons à tirer de la réévaluation de la stratégie de l'OCDE pour l'emploi, Paris, OCDE. 
PIRAT E. (2006), «Sécurisation des parcours professionnels. Négocier de nouveaux droits», disponible sur le site: www.cfdt.fr, 17 mars 2006.

Supiot A. (sous la direction de) (1999), Au-delà de l'emploi. Transformation du travail et devenir du droit $d u$ travail en Europe, Paris, Flammarion.

Travail ET EMPLoI (2008), «Flexicurité et réforme du marché du travail», $\mathrm{n}^{\circ} 113$.

VERDIER E. (2008), «Vers une gouvernance territoriale des risques de l'emploi et du travail», Travail et Emploi, $\mathrm{n}^{\circ} 113$, p. 103-115.

De Virville M. (2004), Pour un Code du travail plus efficace, rapport au ministre des Affaires sociales, de l'Emploi et de la Solidarité, Paris, La Documentation française.

WATt A. (2006), "Assessing the reassessment of the OECD Jobs Strategy: eppur si muove?", European Economic and Employment Policy Brief, № 2. 